\title{
ISPA WJCR
}

\section{Older adults and adult children: Intergenerational relationships and ambivalence}

Sofia von Humboldt \& Isabel Leal

William James Center of Research, ISPA - Instituto Universitário

Aims

The relationship of older adults with their adult children involves great emotional complexity and the quality of these relationships is associated with older adults' well-being. This qualitative study aims to examine how older adults conceptualize intergenerational relationships with adult children.

\section{Methods}

The present study on qualitative data collected from in-depth interviews was conducted with English and Portuguese older adults living in the community, designed to address their perspectives on intergenerational relations with adult children. 316 older adults participated in our study. The mean age of this group was 71.2 years. $65.3 \%$ were women, and a majority (54.7\%) had a partner.

\section{Conclusions}

Intergenerational relationships are experienced by older adults with ambivalence and and stress the contradictory expectations of older adults with grandchildren.

\section{Results}

Content analysis generated four themes: affection and integration; satisfaction in the relationship; privacy and boundaries; financial support.

\section{Conceptualizing support}

1. Older adults-adult children interaction quality: "I am proud of the quality of the relationship with my children. We only see each other twice a year but we have long and profound conversations, I love those moments."

2. Family integration: "I like it when my son asks me to participate in his free time."

3. Care and support: "My mother gave me a lot of affection when I was younger, she helped me to make the most important decisions of my life and I'm very grateful for that. Now it's my turn to give back all the love I've received to my children."

\section{Conceptualizing conflict}

1. Definition of limits "I understand that I have limitations, but I will not stop doing the things I like!"

2. Distance and alienation: "Since my wife died, I began to isolate himself and deprive himself of social activities. My daughter tries to share her life with me but I prefer to stay alone."

3. Communication difficulties : "It may be easy for you, click here and there but for older adults it is very confusing. I feel I am outdated and that I cannot share more of my daughter's life because of this limitation." 